\title{
Foreword to the Special Issue on Laser and optical technologies in biomedicine and ecology
}

We are pleased to present the Special Issue of the Journal of Biomedical Photonics \& Engineering, which focuses on laser and optical technologies for biomedical and ecological applications. This Special Issue consists of the selected papers of the Workshop "Biophotonics" of the XIV allRussian Youth Samara conference-contest on optics and laser physics (Samara, November 8-12, 2016).

The XIV Youth Samara conference-contest on optics and laser physics was organized by Samara Branch of P.N. Lebedev Physical Institute of Russian Academy of Sciences and Samara National Research University with support of Russian Foundation for Basic Research (grant 16-32-10320), Administration of Samara Region and Samara SPIE Student Chapter. The conference program included lectures of leading scientists, reports of young $\mathrm{PhDs}$ and contest reports of students, graduate students and young researches on wide range of theoretical and experimental issues of coherent, physical and quantum optics, spectroscopy, the interaction of light with matter, nanophotonics and other contemporary topics of optics and laser physics. Many interesting reports on applications of laser and optical techniques in biomedicine and ecology were presented at the Workshop "Biophotonics". The Special issue of JBPE will introduce you to some of them.

Laser speckle contrast imaging (LSCI) is a perspective noninvasive, contactless technique for visualization of capillary blood flow in a real-time without laser beam scanning. Polina A. Timoshina and co-authors used LSCI for study of the change in blood microcirculation of the pancreas in rats with alloxane-induced diabetes and under influence of optical clearing agent "Omnipaque TM -300". The authors demonstrated that Omnipaque TM -300 caused changes in the blood flow in the pancreatic vessels only in diabetic mice and suggested that this fact allowed using this clearing agent for monitoring of certain complications caused by some diseases.

Alloxane-induced diabetes of rats was also studied by Daria K. Tuchina and co-authors. In particular, the authors carried out the comparative analysis of glycerol diffusion rate in skin and myocardium of rats in normal condition and under the conditions of model alloxan-induced diabetes. The changes in diffusion rate of glycerol clearing agent reflects the changes of tissue structure and can be usedas a biomarker.

The Issue includes two papers of the researchers from Irkutsk Technical National University and Irkutsk State Medical University: Alexander N. Malov and co-authors. In the first study the experimental research of influence of laser radiation on the bacteria's growth dynamics in the nutrient medium as well as on the antibiotics activity is presented. The authors propose that the decrease in the colonies growth or the increase of the antibiotics efficiency under the laser radiation impact occurs due to the fact that the laser radiation breaks the molecules clusters of the organic solution or of the antibiotic accordingly. And in the second paper on the basis of published data and their own experimental results A. Malov and co-authors suggest possible mechanisms of the formation of gallstones and effect of laser radiation on their growth.

The paper of Vladislav S. Krasnoukhov and co-authors has an ecological focus. The authors studied the reaction between cyclopentadienyl and methyl radicals. The practical importance of the study is determined by the fact that this reaction is a potential source of polycyclic aromatic hydrocarbons having a large negative impact on the environment and human health. The authors found the reaction paths, clarified the energies of compounds and obtained the rate constants of some reactions.

The research by Anastasiya N. Markina and Aleksandr A. Fedotov is focused on the development of new approaches for diagnostic of arterial vessels. The authors suggest that the signal analysis of the pulse wave is noninvasive,relatively cheap and safe diagnostic technique and so it can be used as the most suitable method of screening diagnostics of atherosclerosis. 
The paper of Natalia S. Ksenofontova and co-authors is devoted to investigation of optical immersion clearing of laboratory animal's skin in vivo. A comparative analysis of the changes in the optical depth of the skin detection at preliminary laser ablation of epidermis, the application of immersion agent and the combination of these two techniques is provided.

The principal possibility of the selection of lung cancer samples among the tumors of other localizations by means of Raman spectroscopy and autofluorescent analysis was studied by Ludmila A. Shamina and co-authors. Raman and autofluorescence spectra of blood and urine from patients with cancers of different localization were obtained and analyzed with PLS method. The authors demonstrated that simultaneous study of two human body fluids allows increasing informativeness of the analysis.

The study by Anastasiya A. Lykina and co-authors is directed on the development of nonresonant techniques of Raman spectroscopy for albumin analysis. The authors demonstrated that it is possible to increase the registration efficiency of Raman scattering spectra using special cuvettereflectors of a certain shape. The quantitative characteristics of low albumin's concentrations with dependence on the shape and geometry of the cell were determined, which is very important for development of justified recommendations for biomedical applications.

Nikolay P. Kozlov and Rafael I. Fairushin used the Fourier spectroscopy technique for research of the oxidative degradation of polypropylene under the impact of ultraviolet radiation. The object of study is a polypropylene mesh, which is widely used in medicine. The presence of through-holes in the mesh allows passing only a part of the IR radiation onto the sample determining the features of processing spectra of such meshes.

Thus, the selected papers presented in this Special Issue, introduce you to the major current issues discussed at the workshop "Biophotonics" of the XIV All-Russian Youth Samara conference-contest on optics and laser physics.

Special issue Editors:

Aleksandra M. Mayorova, Samara Branch of P.N. Lebedev Physical Institute of Russian Academy of Sciences

Valery P. Zakharov, Samara National Research University 